\title{
Evaluation of DTN Routing Protocols in Post Disaster Scenario
}

\author{
Nikhil Gondaliya \\ G H Patel College of Engineering \& Technology \\ Vallabh Vidyanagar, Guarat, \\ Vallabh Vidyanagar, India
}

\author{
Shital N Gondaliya \\ Madhuben and Bhanubhai Patel Institute of \\ Computer and Communication Technology, \\ New Vallabh Vidyanagar, Gujarat, India
}

\begin{abstract}
Delay Tolerant Networking (DTN) can be used as a way of communication when existing infrastructure is totally damaged or not possible to establish in the regions like military, disaster and fire fighting. It is most suitable to the application of disaster scenario because end to end path may not be available most of time and connectivity between rescue team member and survivors become opportunistic. Post disaster scenario where it is not possible to predict the movement of people because there is not real trace data available. Some authors represented mobility model in disaster scenario using which it is possible to generate movement pattern of rescue team members and survivors. One of the factors which affect the performance of DTN is design of routing protocol. Many DTN routing protocols have been proposed in the literature but their performance depends mainly on the application where it is used. In this paper, we explore the performance of five different routing protocols namely Epidemic, Binary Spray and Wait (BSnW), Spray and Focus (SnF), Encounter Based Routing (EBR) and PRoPHET. We have evaluated performance of these protocols in terms of delivery probability, overhead ratio, average delay and average hop count by varying buffer size, message size and number of messages per minute. Simulation is conducted using Opportunistic Network Environment (ONE) simulator.
\end{abstract}

\section{Keywords}

Delay tolerant network, disaster network, routing protocol

\section{INTRODUCTION}

Natural disasters like earthquake, fire fighting or tsunami disorder human activity and disconnects communication services like phone and the Internet. Existing infrastructure is damaged by disaster and fundamental challenge is to provide communication between victim and rescue team members (fire fighter, police and ambulances). DTN is promising communication model in wireless communication network which enables communication without need of fixed infrastructure. Traditional wireless network where network partition is rare and end to end path between sources to destination is available most of the time. DTN employs "store and forward" approach of data transfer, where data is sent hop by hop and stored at intermediate nodes until node has an appropriate contact opportunity to pass it. The nodes of DTN are smartphone and laptop which can support communication technology such as Bluetooth or Wi-Fi. We assume that people are carried with DTN enabled device with Bluetooth technology to support communication in disaster network.
The paper is organized as follows. Section II presents related work. Section III discusses disaster scenarios. Section IV explains brief on DTN routing protocol. Section V shows simulation setup and results. Finally, Section VI concludes the paper with future direction.

\section{RELATED WORK}

In the past many disaster occurred and few countries are facing the problem of earthquake and tsunami very frequently. In these events, the communication infrastructure (cellular network, satellite network) is partially or totally damaged for many days. Researcher proposed solution to provide communication and collect victim data in emergency situation using their owned network or other way. McGrath et al. [1] proposed system that provides automated remote triage and emergency management information through the use of sensors. Triage information is transmitted using agents that move through a reliable messaging layer in wireless ad hoc networks. Lorincz et al. [2] recommended a system that uses sensors to track victims and monitor their crucial signs. The transmission of data is done using wireless sensor networks created by the sensors deployed in the emergency area. Williams [3] proposed a system for military to capture and display real-time casualty data in the field where a handheld unit stores the casualty data and the GPS position and uses satellite communication to send it.

Some authors have attempted to model mobility in disaster situation and generated synthesis traces which mimic to post disaster state. These traces are tested in Mobile AdHoc NETwork (MANET) and DTN environment.

Samuel et al. [4] proposed event-driven role-based mobility model to predict the movement of objects (civilians, police, fire fighter, ambulance, hospital) in post disaster situation using physics based gravitational model. When event occurs, some objects (police, fire fighter and ambulances) are attracted towards event and some (civilian) are flew from events with force which is calculated based on distance from the event and intensity of event. They also tested their mobility model in DTN environment and devised Encounter Based Routing (EBR) protocol [5] for DTN which performs well under movement of objects as prescribed in their disaster recovery network model.

$\mathrm{N}$ Aschenbruck et al. [6] modeled mobility in disaster area scenario which based on separation of rooms. Disaster area is divided into different rooms like incident location, patient waiting area, causality clearing station, ambulance parking area and technical operation command. Object (human or ambulance) behaves differently based on their role and moves 
between or inside room. They analyzed characteristics which influence network performance in public safety communication networks like heterogeneous area-based movement, obstacles, and joining/leaving of nodes which cannot be modeled using existing mobility model. A. Mart'inCampillo et al. [7] evaluated performance of existing routing protocol for this disaster area scenario under DTN environment by considering two locations namely incident and patient waiting area. It is assumed that patient waiting area is connected through internet or other means to transfer data to some central location.

Md Y. S. Uddin et al. [8] presented a post disaster mobility model for delay tolerant networking to provide communication in contexts where it is difficult to assume end to end connectivity. Their mobility model comprises the impact of disaster on the transportation network, population and relief vehicle movement.

\section{DISASTER SCENARIO}

Modeling mobility for disaster scenario is impractical because no real time traces are available to predict the movement of rescuer and victims. Even mobility of mobile objects varies based on type of disaster. In some disaster, population is forewarned (tsunami) and while in other population shift happens (earthquake) after event.

Table 1: Disaster Scenario Parameters

\begin{tabular}{|l|l|}
\hline \multicolumn{1}{|c|}{ Parameter } & \multicolumn{1}{c|}{ Value } \\
\hline Number of Civilians & 100 \\
\hline Number of Police/Fire fighter & 50 \\
\hline Number of Ambulances & 50 \\
\hline Number of Hospitals & 4 \\
\hline Number of Events & 4 \\
\hline
\end{tabular}

We have chosen event-role based mobility model [4] to evaluate the performance of routing protocol. There are four types of objects in this model namely civilians, policeman/fire fighters, ambulance and hospital. When event occurs object behaves differently based on the role assigned to them. There are four hospitals which are immobile and located on the border area. Ambulances are assigned to specific events and they move back and forth between hospitals when event takes place. Civilians move within the simulation area randomly (change direction every $30 \mathrm{Sec}$.) and reacts to event and fly away from the events based on the intensity of event if they are within event damage or horizon radius. Each event has associated intensity which is in the range of 10 to 20 thousands. Police or fire fighters are attracted to events as they are rescue members based on the gravitational force which is calculated as intensity of event divided by square of distance between them. Table 1. shows the configuration parameter used to generate disaster scenario.

\section{ROUTING PROTOCOLS}

DTN routing protocols are broadly classified in forwarding based or flooding based depending whether they create message replica or not. Routing protocols that maintain single copy of message is called forwarding based routing protocol where those create more than one copy of message are called flooding or replication based routing protocol. We have evaluated performance of five different flooding based existing routing protocols namely Epidemic, EBR, PRoPHET, BSnW and SnF.

\subsection{Epidemic routing protocol}

Epidemic routing protocol [9] guarantees that each node will eventually receive the message copy. Each node maintains summary vector which is used to keep the track of messages they have. When nodes meet each other then they exchange the summary vector and transfer the message they do not have. This protocol has maximum delivery probability and minimum delay but at the cost of routing overhead.

\subsection{Encounter Based Routing protocol}

EBR [5] protocol which observes mobility pattern from many networks: a future rate of encounter can be roughly predicated from past data. Node which encounter more frequently is likely to pass messages to destination successfully than those who infrequently encounter others. This protocol is quota based where copy of message replica is controlled by node's encounter rate. Number of copies to be given when node encounter to other node is based on encounter value of that node. EBR maximizes delivery ratio and minimizes average delay and overhead than other flooding based protocols: BSnw, Epidemic and SnF under two different mobility model a tradictional Random Way Point (RWP) and disaster scenario [4].

\subsection{PRoPHET}

PRoPHET [10] in which every node maintains delivery predictability value $([0,1])$ for known destination which indicate how likely it is that this node will be able to deliver a message to that destination. Otherwise operation of PRoPHET is similar to Epidemic routing. When two nodes meet, they exchange summary vectors which in this case also contain the delivery predictability information stored at the nodes. This information is used to update the internal delivery predictability value and then the information in the summary vector is used to decide which messages to request from the other node based on the forwarding strategy used.

\subsection{Binary Spray and Wait}

BSnW [11] which works in two phases; the Spray phase and Wait phase. In the Spray phase, source node generates L copies of the message and sprays it to $\mathrm{L}$ different encountered nodes. If node is having more than one copy of message left will gives half of the copies and keeps the remaining to itself until one copy is left. In Wait phase, if destination is not found in spraying phase then each of the node which is having a single copy of the message performs direction transmission. This protocol controls the number of replicas of message compared to Epidemic protocol and hence less overhead but problem is to choose number of copies of message to spray in the network.

\subsection{Spray and Focus}

SnF [12] routing protocol also operates in two phase; the Spray phase which is similar to BSnW and the Focus phase. Unlike Spray and Wait, where in the Wait phase messages are routed using Direct Transmission, in the Focus phase a message can be forwarded to a different relay according to a specific forwarding criterion. 


\section{SIMULATION RESULT}

We have chosen simulation methodology to analyze the performance of existing routing protocols in disaster scenario using ONE simulator 1.4.1 [13].

\subsection{Performance Metrics}

The metrics that are chosen to analyze the performance of routing protocols in disaster scenario are:

Delivery Ratio: It is ratio of number of message delivered successfully to that of total number of message created.

Average Delay/Latency: Latency is time elapsed between creation of message at source to its delivery at destination. Average delay is total of latencies of all message delivered successful.

Overhead Ratio: The Overhead Ratio is used to measure the number of redundant message relayed to transmit one message to its destination. It is given by following equation.

$$
\text { Overhead Ratio }=\frac{(R-D)}{D}
$$

Where $R$ is number of message relayed and $D$ is number of message delivered to the destination successfully

Average Hop Count: It is the average of number of hop count between each source-destination pair for delivered messages.

\subsection{Simulation Parameters}

Simulation parameters which are common for all simulation are listed in Table 2. It is assumed that people are carrying with hand held DTN enabled device with them in order to support the communication in disaster area.

\subsection{Results and Discussion}

Routing protocol performance is evaluated for above metrics in disaster scenario for five routing protocols by varying buffer size, message size and message generation rate.

Table 2: Simulation Parameters

\begin{tabular}{|l|l|}
\hline \multicolumn{1}{|c|}{ Parameter } & \multicolumn{1}{c|}{ Value } \\
\hline Simulation Area Size & $3000 \mathrm{~m} * \mathrm{~m}$ \\
\hline Simulation Time & $2000 \mathrm{Sec}$ \\
\hline Number of Nodes & 204 \\
\hline Interface & Bluetooth \\
\hline Transmission Range & $250 \mathrm{~m}$ \\
\hline TTL & $50 \mathrm{~min}$. \\
\hline
\end{tabular}

Impact of Varying Buffer Size

Buffer size is varied from $5 \mathrm{M}-60 \mathrm{M}$ in the interval of $5 \mathrm{M}$. Result in Fig. 1 shows that delivery ratio for Epidemic and PRoPHET protocol is maximum and remain constant at buffer size 35M. Whereas EBR, BSnW and SaF do not have effect of buffer size on delivery ratio, they perform similar irrespective of buffer size. Fig. 2 indicates that Epidemic protocol has lowest average delay than others and becomes steady at buffer size $35 \mathrm{M}$.

Table 3: particular Parameters

\begin{tabular}{|l|l|}
\hline \multicolumn{1}{|c|}{ Parameter } & \multicolumn{1}{c|}{ Value } \\
\hline Message Size & $256 \mathrm{~K}$ \\
\hline Buffer Size & $5 \mathrm{M}-60 \mathrm{M}$ \\
\hline Message Interval Rate & At every $10-20 \mathrm{sec}$ \\
\hline
\end{tabular}

Fig. 3 represents that Epidemic and PRoPHET protocol have more overhead in the beginning and decreases as buffer size increases and becomes stable at buffer size $35 \mathrm{M}$. While other three protocols have lower overhead irrespective of buffer size. Fig. 4 shows that average hop count remain invariable at any buffer size for EBR and BSnW. Avarage hop count fluctuate slightly for $\mathrm{SnF}$ and PRoPHET protocol. Metric value is increase as buffer size increases in Epidemic protocol but becomes fixed at $35 \mathrm{M}$.

It is observed that buffer size $35 \mathrm{M}$ is sufficient for Epidemic protocol to attain reasonable delivery ratio, minimum average delay and minimum overhead where others do not have much effect of varying buffer size.

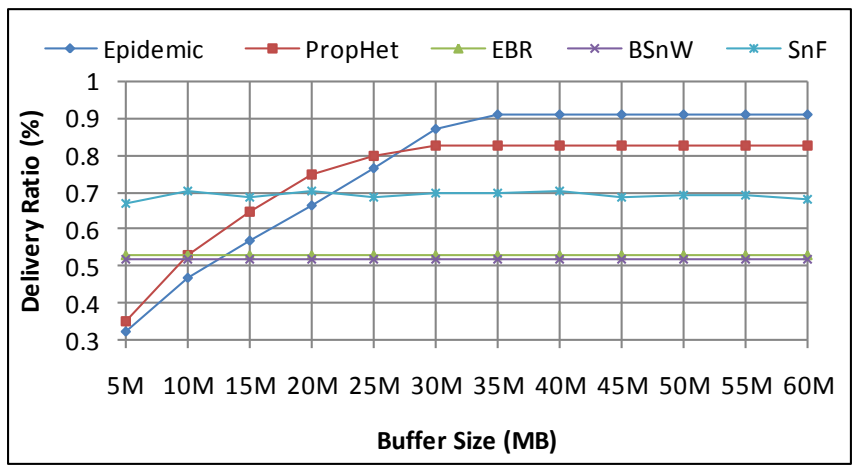

Fig. 1. Buffer size Vs delivery ratio

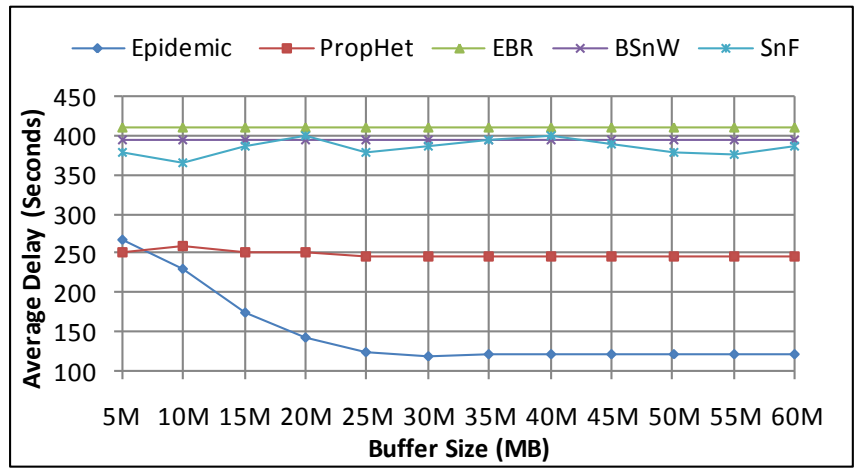

Fig. 2. Buffer size Vs average delay

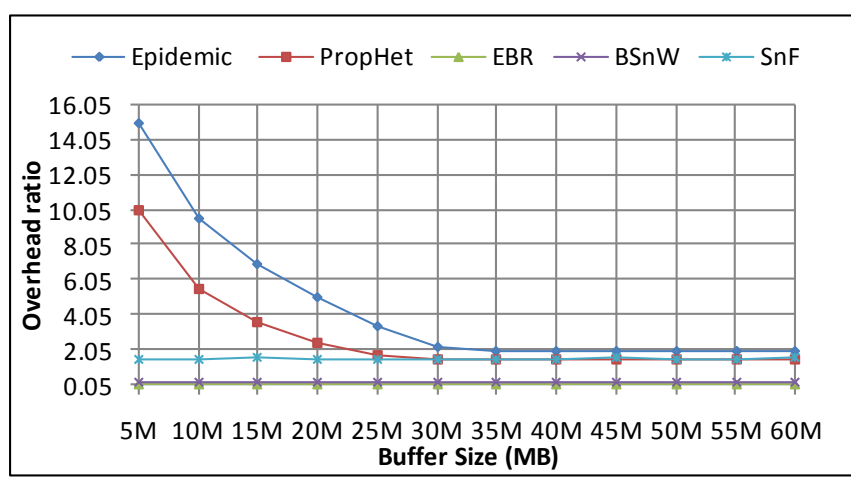

Fig. 3. Buffer size Vs overhead ratio 


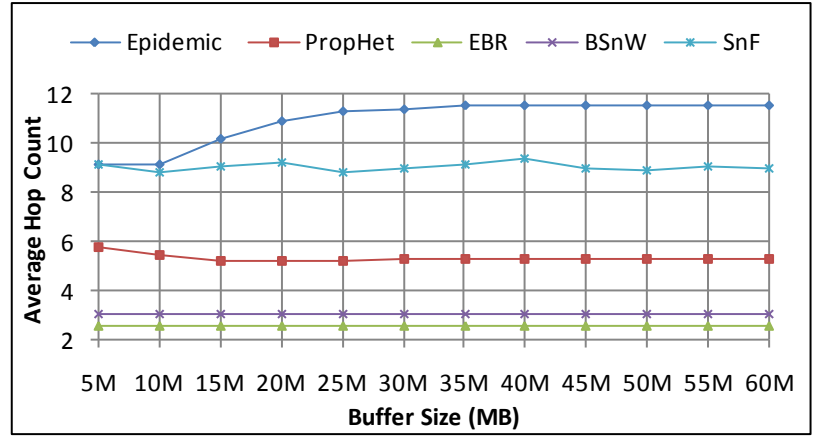

Fig. 4. Buffer size Vs average hop count

Impact of Varying Message Size

In this simulation, message size is varied from $64 \mathrm{~K}$ to $7 \mathrm{M}$ and analyzed the effect on performance of routing protocol.

Table 4: Particular Parameters

\begin{tabular}{|l|l|}
\hline \multicolumn{1}{|c|}{ Parameter } & \multicolumn{1}{c|}{ Value } \\
\hline Buffer Size & $35 \mathrm{M}$ \\
\hline Message Size & $64 \mathrm{~K}-7 \mathrm{M}$ \\
\hline Message Interval Rate & At every $10-20 \mathrm{Sec}$. \\
\hline
\end{tabular}

Fig. 5 shows that Epidemic and PRoPHET have good delivery ratio up to message size of $256 \mathrm{~K}$ then it starts decreasing. Delivery ratio is steady up to $1 \mathrm{M}$ for $\mathrm{SnW}$ protocol and then begins to decreases. Whereas for others, there is not any effect of changing message size on delivery ratio. Figure 6 illustrates that Epidemic and PRoPHET protocol have minimum average delay compared to others. It remains stable up to $256 \mathrm{~K}$ and $512 \mathrm{~K}$ respectively for Epidemic and PRoPHET then starts increasing. EBR, BSnW and SnF protocol do not have much effect of message size on average delay.

Fig. 7 demonstrates that overhead is steady up to message size of $256 \mathrm{~K}$ for Epidemic and PRoPHET protocol then it increases. Overhead remain stable for other protocols and not affected by message size. Fig. 8 shows that average hop count is stable till $256 \mathrm{~K}$ and $1 \mathrm{M}$ message size for Epidemic and $\mathrm{SnF}$ protocol respectively.

It is examined from the result that $256 \mathrm{~K}$ message size is sufficient for Epidemic and PRoPHET protocol where other protocols do not affected by message size.

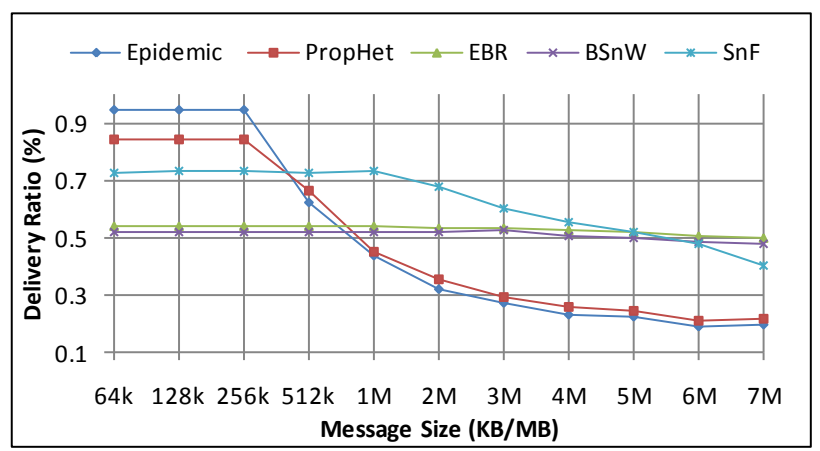

Fig. 5. Message size Vs delivery ratio

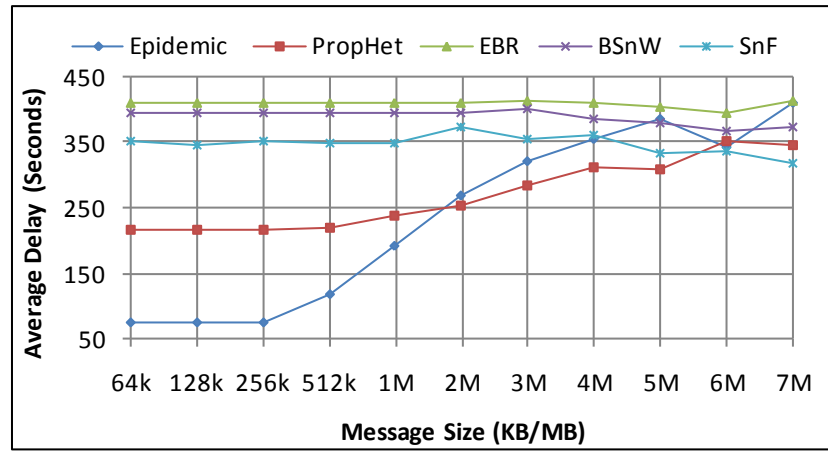

Fig. 6. Message size Vs average delay

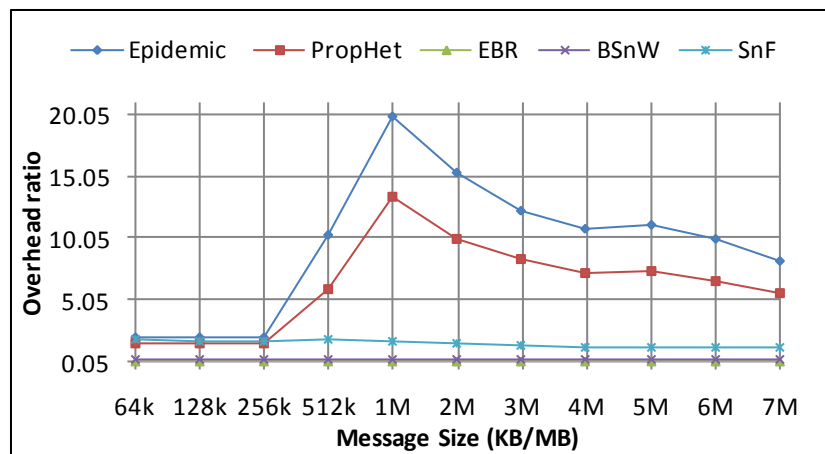

Fig. 7. Message size Vs overhead ratio

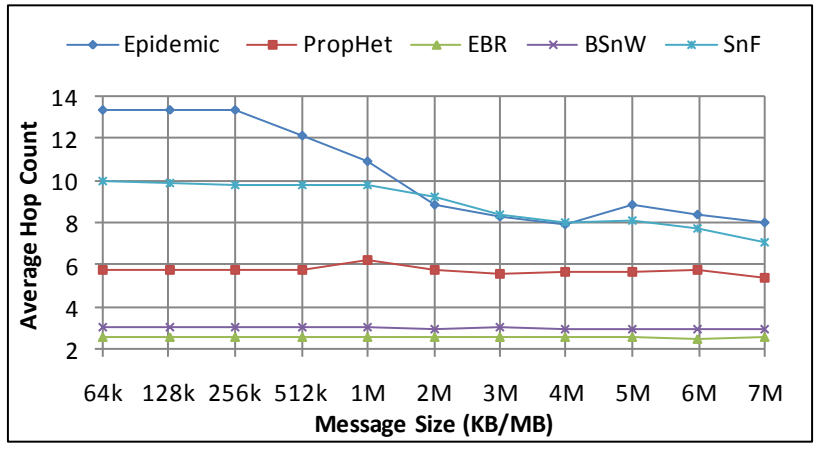

Fig. 8. Message size Vs average hop count

\section{Impact of Varying Traffic Rate}

We have also analyzed the effect of varying message generation rate means number of message generated per minute. Figure 9 depicts that delivery ratio increase as message interval size decreases for Epidemic, PRoPHET and SnF protocol while for BSnW and EBR it remain same and stable for any message size. There is no effect of varying traffic flow on average delay for PRoPHET, EBR, SnF and BSnW protocol as shown in Fig. 10. While Epidemic protocol has maximum average delay and starts decreasing as traffic flow decreases.

Table 5: Particular Parameters

\begin{tabular}{|l|l|}
\hline \multicolumn{1}{|c|}{ Parameter } & \multicolumn{1}{c|}{ Value } \\
\hline Buffer Size & $35 \mathrm{M}$ \\
\hline Message Size & $256 \mathrm{~K}$ \\
\hline & At every 1-2, 2-3, 3-4, \\
Message Generation & $4-5,5-6,6-7,7-8,8-9$, \\
Interval & $9-10,10-11,11-12$ \\
& (Sec) \\
\hline
\end{tabular}


Fig. 11 shows that overhead ratio is consistent and lowest for EBR and BSnW protocol irrespective of traffic flow. It is increased as traffic flow decreases for PRoPHET and Epidemic protocol till message generation interval 6-7 then starts decreasing. Average hop count is smallest and stable for BSnW and PRoPHET protocol while it increases as traffic flow decreases as depicted in Fig. 12.

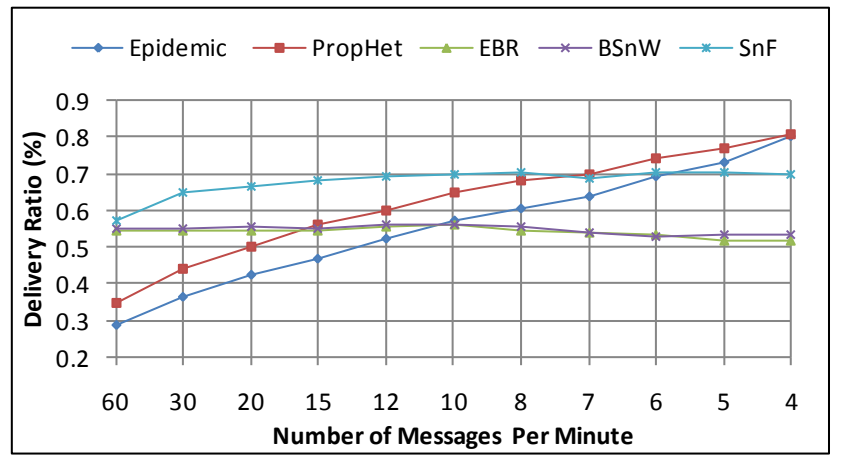

Fig. 9. Message generation rate Vs delivery ratio

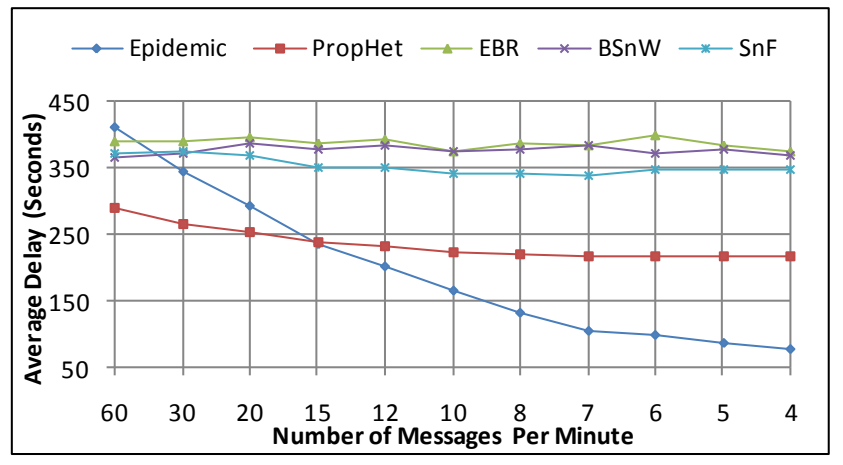

Fig. 10. Message generation rate Vs average delay

It is viewed that BSnW performs better in terms of delivery ratio for even high traffic flow in the network compared to others. PRoPHET has minimum average delay and smaller average hop count than other routing protocol for high as well low traffic flow.

\section{CONCLUSIONS AND FUTURE DIRECTIONS}

In this paper, we have evaluated the performance of five flooding based DTN routing protocol in disaster scenario by varying various parameters like buffer size, message size and traffic intensity. Movement pattern of rescue team and victim is not possible to predict as real time traces are not available. So, we have chosen event-role based mobility model to generate the synthetic traces which imitate the post disaster scenario. We have found out the optimum size of different parameters for routing protocols. Buffer size of $35 \mathrm{M}$ is sufficient for Epidemic and PRoPHET routing protocol to achieve maximum delivery ratio, minimum average delay and minimum overhead ratio where others do not have effect of buffer size. Epidemic and PRoPHET performs better in terms of delivery ratio and average delay up to message size of $256 \mathrm{~K}$ while $\mathrm{SnF}$ achieves the same till size of $1 \mathrm{M}$. EBR and BSnW have same delivery ratio irrespective of message size. Delivery ratio increases and average delay decreases as traffic intensity decreases for Epidemic and PRoPHET while others do not much change in it.

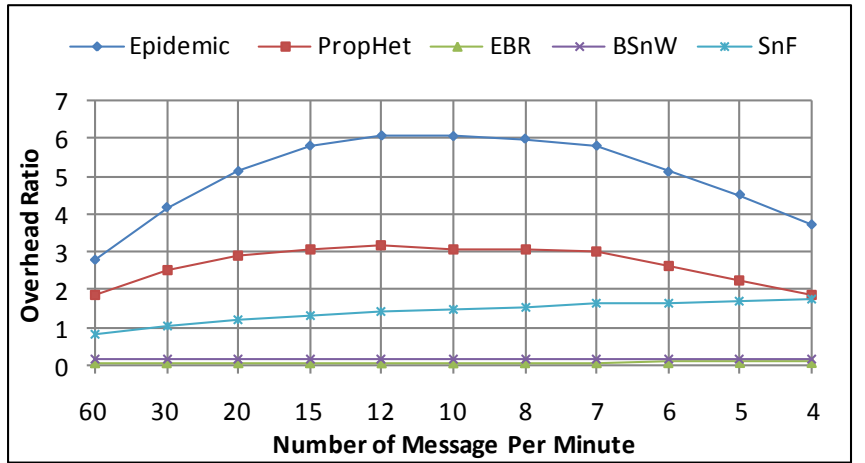

Fig. 11. Message generation rate Vs overhead ratio

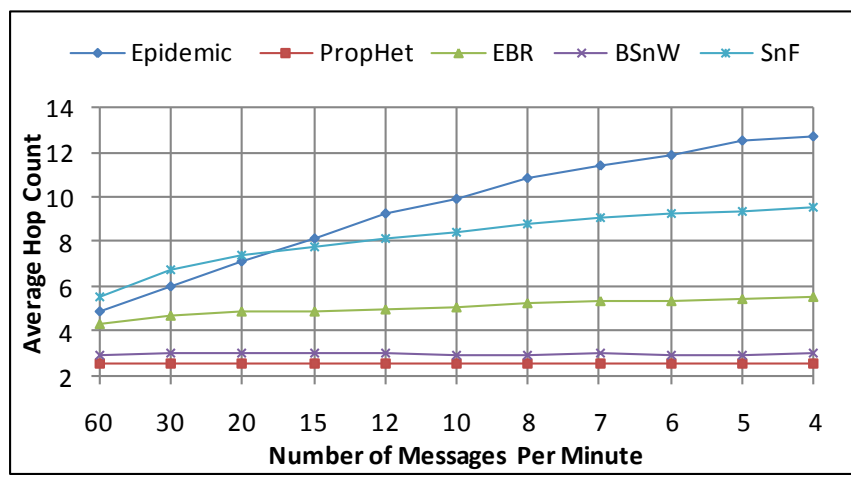

Fig. 12. Message generation rate Vs average hop count

Mobility models proposed till date have considered random movement of human using either Random Walk or Random Way Point model. In realistic, human mobility is not random but they move with some feature which can be taken in to consideration as future work. One can also add obstacles (buildings, homes etc...) that remain present mostly in the disaster area which is not part of even role based mobility model. Another future direction is to design DTN routing protocol especially for disaster scenario to achieve desirable maximum delivery ratio and minimum average delay.

\section{REFERENCES}

[1] McGrath S, Grigg E, Wendelken S, Blike G, Rosa MD, Fiske A, et al. ARTEMIS: a vision for remote triage and emergency management information integration. Dartmouth University; 2003. p. 9.

[2] Lorincz K, Malan D, Fulford-Jones T, Nawoj A, Clavel A, Shnayder V, et al. Sensor networks for emergency response: challenges and opportunities. Pervasive Computing, IEEE 2004;3(4):16-23.

[3] Williams D. Tactical medical coordination system (tacmedcs), Naval Health Research Center, San Diego, CA. Technical report. Febraury 2004-June 2007; November 2007

[4] Samuel C. Nelson, Albert F. Harris, and Robin Kravets. Event-driven, role-based mobility in disaster recovery networks. In CHANTS, 2007

[5] Samuel C. Nelson, M. Bakht and R. Kravets, "Encounter-Based Routing in DTNs," Proceedings of IEEE INFOCOM, Rio De Janeiro, Brazil, April 2009.

[6] N. Aschenbruck, E. Gerhards-Padilla, M. Gerharz, M. Frank, P. Martini, Modelling mobility in disaster area scenarios, in: Proceedings of the 10th ACM Symposium 
on Modeling, Analysis, and Simulation of Wireless and Mobile Systems, MSWiM'07, ACM, New York, NY, USA, 2007, pp. 4-12.

[7] A. Mart'ın-Campillo, J. Crowcroft, E. Yoneki, R. Mart'1, Evaluating opportunistic networks in disaster scenarios, Journal of Network and Computer Applications 36 (2) (2013), pp. 870-880.

[8] M. Y. S. Uddin, H. Ahmadi, T. Abdelzaher, and R Kravets. A low-energy, multi-copy inter-contact routing protocol for disaster response networks. In Proc. of IEEE SECON, NJ, USA, 2009, pp. 637-645.

[9] A. Vahdat and D. Becker, "Epidemic routing for partially connected ad hoc networks," Tech. Rep., Duke University, Durham, NC, USA, 2000.

[10] A. Lindgren, A. Doria, and O. Schelén, "Probabilistic routing in intermittently connected networks," ACM
SIGMOBILE Mobile Computing and Communications Review, vol. 7, no. 3, 2003, pp. 19-20.

[11] T. Spyropoulos, K. Psounis, and C. Raghavendra, "Spray and wait: An efficient routing scheme for intermittently connected mobile networks," in Proc. WDTN, 2005.

[12] T. Spyropoulos, Konstantinos Psounis, and Cauligi S. Raghavendra. Spray and focus: Efficient mobilityassisted routing for heterogeneous and correlated mobility. In Fifth Annual IEEE International Conference on Pervasive Computing and Communication Workshops, 2007.

[13] Keränen, A., Ott, J., and Kärkkäinen, T, "The ONE Simulator for DTN Protocol Evaluation", In Simutools '09: Proceedings of the 2nd International Conference on Simulation Tools and Techniques (2009), ICST (Institute for Computer Sciences, Social-Informatics and Telecommunications Engineering), pp.1-10. 